This is an Accepted Manuscript of an article published by Taylor \& Francis in Cataloging \& Classification Quarterly on 21 Dec. 2015, available online:

http://www.tandfonline.com/10.1080/01639374.2015.1105898.

\title{
Popular Music in FRBR and RDA: Toward User-Friendly and Cataloger-Friendly Identification of Works
}

\section{Kevin Kishimoto (University of Chicago) \& Tracey Snyder (Cornell University)}

\begin{abstract}
:
The gradual adoption since 2010 of the content standard Resource Description and Access, based on the conceptual model Functional Requirements for Bibliographic Records, has brought change to many areas of library cataloging, including popular music. In particular, the cataloging community has had to grapple with new practices in assigning access points for resources once considered simple, such as popular music albums containing songs written by people other than the featured recording artist. This article outlines some of the difficulties encountered and offers a principled approach to cataloging popular music that would reduce cataloger burden and reconcile catalog data with users' expectations.
\end{abstract}

\section{INTRODUCTION}

Who can forget the deliciously awkward scene in the 2001 romantic comedy Bridget Jones's Diary in which the hapless title character performs a badly out-of-tune rendition of the classic ballad "Without You" at a party at the office, with a smoking cigarette in her hand and at least a few drinks in her system? If you grew up in, or were at least present for, the 1970s, you probably recognized this as the late Harry Nilsson's hit single from 1971. If you were young in the 1990s, you may instead have recognized this as Mariah Carey's hit single from 1994. If both of these singles predate your musical awareness, perhaps you know this song from performances on American Idol by the likes of Kelly Clarkson or Clay Aiken. But it's probably only enthusiasts of British power pop who would identify this 
as a Badfinger song from 1970, let alone be able to name its composers-Pete Ham and Tom Evans of Badfinger. Although they composed the music and lyrics of the song, the typical listener would likely identify the various performers as the artists responsible for the vastly different versions spanning different eras, from Badfinger's understated performance to the melodramatic ones that followed. Despite this, when using a library catalog, the listener would find a single heading representing all versions of this song, no matter who the performer is:

\section{Ham, Pete. Without you}

Under the current North American implementation of the cataloging standard Resource Description and Access (henceforth, RDA), a single musical work can be represented by an authorized access point (henceforth, AAP) constructed by combining (in simplified terms) the name of the composer with the title of the composition. Such an AAP would make perfect sense in a bibliographic record for a musical score of this song such as the one copyrighted in 1970 and published in 1994 after the song was popularized by Mariah Carey, which reproduces the lyrics and vocal melody and supplies a piano accompaniment and guitar chords. However, this AAP, if it were to be included in a bibliographic record for an audio recording of Mariah Carey's rendition of the song, would only tell part of the story. Popular music artists such as Nilsson, Carey, or any of this song's latter-day interpreters do much to bring a composition to life, making any number of creative decisions along the way and thereby cementing the notion in the minds of listeners that this is their song.

How does this dissonance between composers and performers play out at the album level? In RDA, ${ }^{1}$ which is based on the entity-relationship model known as $\mathrm{FRBR},{ }^{2}$ catalogers are made to consider the authorship of each individual song in making a determination of how to treat the album. In a library catalog, the RDA AAP for Harry Nilsson's album containing the song "Without You" would be:

\section{Nilsson Schmilsson}

And Mariah Carey's album could be:

\section{Music box (1993 : Columbia Records, Inc.)}


This is because the artists did not compose all of the songs on their respective albums. The previous cataloging standard $\mathrm{AACR} 2^{3}$ provided special exceptions in determining main entry for some resource types, such as sound recordings of popular music. AACR2 21.23C1 and 21.23D1 allowed, under most common circumstances, the "principal performer" to serve as the main entry for an album-Harry Nilsson's and Mariah Carey's albums were entered under their names even though they are not the composers of all of the songs contained in these albums:

\section{Nilsson, Harry. Nilsson Schmilsson}

\section{Carey, Mariah. Music box}

Since both of these albums originated before the existence of RDA, a user could conceivably see both types of headings in the catalog for each album, a confusing situation if there ever was one.

Even ignoring the bibliographic records created under the earlier standard, different albums in an artist's oeuvre may receive different treatment in RDA. For example, Harry Nilsson wrote all of the songs on his album Knnillssonn, so the AAP for that album, by contrast with Nilsson Schmilsson, would be:

\section{Nilsson, Harry. Knnillssonn}

The works of Nilsson's friends and Badfinger's Apple Records labelmates the Beatles suffer a similar fate at the hand of RDA. The library catalog could contain any number of RDA AAP constructions representing the Beatles' songs and albums (and movies), and not a single one would be built on the AAP representing the band. Looking at the following list of possible RDA AAPs, catalog users would probably be hard-pressed to figure out which works they represent and why they are not uniformly constructed:

Beatles (Beatles : 1968)

Beatles (Beatles : 2009)

Hard day's night (Motion picture)

Help! (Beatles)

Help! (Motion picture : 1965)

Lennon, John, 1940-1980. Hard day's night (Album)

Lennon, John, 1940-1980. Hard day's night (Song) 


\section{Lennon, John, 1940-1980. Help!}

\section{Lennon, John, 1940-1980. Let it be}

\section{Let it be (Beatles)}

\section{Let it be (Motion picture)}

The news is not all bad. RDA allows for a performance of a musical work involving substantial creative responsibility for adaptation, improvisation, etc., on the part of the performer or performers to be considered a new work. This has led some to take a position that jazz performances and recordings should typically be considered adaptations, and therefore new works created by the performers, since improvisation is at the heart of jazz. But why should pop and rock, which is replete with individualized guitar solos, vocal melisma, drum breaks, and more, be treated differently? It can be asserted that any popular musical artist's rendition of a song by definition constitutes an adaptation of the abstract composition.

In this article, we argue for an interpretation of RDA that posits two distinct works where audio recordings of popular music are involved - an underlying composition, like that of Pete Ham and Tom Evans, and a realization of the composition, like that of Harry Nilsson or Mariah Carey. We believe such an interpretation would bring many benefits for both catalogers and end users of the library catalog, including efficiency of data production and alignment with expectations that are both evidenced by and influenced by other data sources on the Internet.

\section{MUSICAL CULTURES}

In FRBR, the concept of the work is one of its most important, yet is also one that is ill-defined: "a distinct intellectual or artistic creation." Through the identification of a work, one is able to "give a name and draw relationships to the abstract intellectual or artistic creation that encompasses all the individual expressions of that work." 5 Since RDA is based on the FRBR model, an important component of the RDA standard are the guidelines for constructing AAPs for works which assign each work a unique identity and presumably allow users to find, identify, and select (and explore) these works through online 
catalogs and discovery tools. RDA gives many explicit instructions for the creation of AAPs, but is not specific in its discussion of what constitutes a work. This nonspecificity actually is rooted in FRBR, which states:

Because the notion of a work is abstract, it is difficult to define precise boundaries for the entity. The concept of what constitutes a work and where the line of demarcation lies between one work and another may in fact be viewed differently from one culture to another. Consequently the bibliographic conventions established by various cultures or national groups may differ in terms of the criteria they use for determining the boundaries between one work and another. ${ }^{6}$

The fact that the defining criteria of "work" can differ from one culture to another, including different musical cultures that coexist within the same geographic culture, is the crux of our argument for change in cataloging popular music in RDA. In actuality, little revision to the RDA standard itself would be required, but rather a shift in perspective, redefining the concept of the work in describing popular music, and some alteration in national best practices.

In the context of this article, the term "popular music" will refer to the large and broadly defined corpus of music in a popular idiom (to borrow language from AACR2 and RDA) from the 20th and 21st centuries, typically performed and recorded for mass consumption, that does not fall under Western art music (colloquially known as "classical music"). "Popular music" genres include rock, pop, soul, blues, country, jazz, gospel, as well as music from some world folk music traditions. The use of the terms "popular music" and "classical music" is not intended to indicate the actual popularity, or lack thereof, of the musics themselves, nor to make judgment on their artistic merits, but simply as widely used and contrapuntal labels to distinguish these two very different musical traditions and cultures.

A side-by-side comparison of the classical music and popular music traditions will make apparent some striking differences between these cultures, so much so that the authors believe that using different cataloging practices in describing these two musical traditions merits consideration. Appendix A of the 
BIBFRAME AV Modeling Study discusses some major differences between these cultures, ${ }^{7}$ upon which we will expound. These are broad generalizations which, of course, have exceptions.

Composers are well-identified on classical music resources, usually displayed in a position of prominence. Except in cases of collaboration, pasticcio, or misattribution, authorship in classical music tends to be straightforward. In the classical music tradition, the composer holds a place of almost deitylike reverence. These composers create fully fleshed out compositions in specific personal artistic styles, the written music usually firmly set once published. Composers are responsible for determining most of the specific musical elements which make up the work: melody, harmony, rhythmic values and durations, exact pitches, exact medium of performance, etc. In performance, classical musicians strive toward faithful interpretation of the written compositions over individualism, so much so that alterations to the musical text, such as unwritten "ornaments" or even a subtle disregard for the composer's own performance practices, could cause a performance to be judged as poor.

By contrast, the names of songwriters are often not prominent on popular music resources, the performers' names being in the marquee position. The songwriters' names are found in a small font, much smaller than that used for the featured performers, discreetly printed in an unassuming place within the resource. The songwriters' names are also often not even given in full, but abbreviated. While there are many famous and well-recognized songwriters in popular music, there are just as many who are unknown to the general public.

While it is possible that a popular music song could be fully fleshed out like those in classical music, this type of composition is not the norm in the popular music tradition. The "composed" songs can be quite skeletal, sometimes consisting of just melody, lyrics, and perhaps a general idea of the chord progression and tempo. Much creative energy is expended in rehearsal or in the studio when musicians decide how to interpret the original song, filling out the musical texture with material invented by the performers, not the songwriters. Meanwhile, producers and engineers take an active role in the creative process, deciding which instrument or voice to highlight and how to create a good musical progression 
over the course of the song's duration. Recording popular music can be a very collaborative process involving many individuals who add to the creative whole.

One of the biggest differences from the classical music tradition is the expectation that popular musicians perform their own creative and individualized interpretations of previously existing songs of which they themselves can take ownership. In fact, these versions often include much original musical material, including altered melodies or chord progressions, changed lyrics, and added instrumentation, and sometimes are performed in a completely different style or genre from that which the songwriter originally intended.

One song that clearly demonstrates differences between variant performed versions is "Proud Mary," a song written by John Fogerty and first recorded by his band Creedence Clearwater Revival. Released in 1969, this song in the roots rock style incorporates elements of folk music, country, and blues. It peaked at \#2 on the Billboard charts and is one of CCR's best-known songs. Two years later, Ike \& Tina Turner released their recording of "Proud Mary," which sounds substantially different from the CCR original. The Ike \& Tina Turner version is performed in a funk rock/soul style, beginning with a soft and slow introduction then quickly launching into a boisterous uptempo section complete with loud horns and gospel-like backing vocals. This cover version has proven to be very successful for the band, reaching \#4 on the Billboard Hot 100, winning the 1972 Grammy Award for Best R\&B Vocal Performance by a Group, and becoming a signature song for the band. This cover song has become so closely identified with this band that a few of their greatest hits albums bear titles like Proud Mary: The Best of Ike \& Tina Turner and Proud Mary and Other Hits. (Readers are encouraged to listen to both CCR's and Ike \& Tina Turner's versions of "Proud Mary," both readily available on the World Wide Web, to observe their profound differences.) Other notable cover versions of "Proud Mary" in which performers adapted the song to their personal artistic styles are: a live recording of Elvis Presley performing the song in the spirited style for which 1970s-era Elvis is known; a very funky version by the Ohio Players; and a recording by the instrumental surf rock band the Ventures. 
Another significant difference between the classical music and popular music traditions is the fact that these traditions differ in their fundamental mechanism of dissemination. Classical music has a longstanding literate tradition, with written music shared through published and manuscript copies. Classical musicians are trained to read symbols on paper and perform the music as instructed. On the other hand, musicians in the popular music tradition have more varied backgrounds-some can read and perform from written music, while others cannot. In fact, one could argue that popular music is more of an oral tradition than a written one. Furthermore, popular music "sound recordings often serve as the primary mode of transmission and common source of reference for people." ${ }^{\prime 8}$ Printed popular music resources, when they exist, are often based on (i.e. transcribed from) the recordings. Because of this fundamental difference between the two traditions, it is not difficult to imagine that the concept of the work could actually refer to music in two very different states: the written composition in classical music and the recorded song in popular music.

The differences between the classical music and popular music traditions extend beyond the composers, songwriters, and performers themselves; the expectations, preferences, and practices of users are also different. Classical music users make heavy use of both scores and recordings, while the vast majority of popular music users will only be seeking recorded music. Our experience as librarians and classical musicians tells us that users of classical scores and recordings tend to focus primarily on composers and their works before all else. The performers on audio recordings, in most cases, would be the second or third factor under consideration. On the other hand, our experience as librarians and popular music fans tells us that users of popular music recordings base their searching primarily on performers and songs or albums. While the song as a composition is important to users, it is usually a specific performer's rendition of the song that is the focus of a search.

One can hardly imagine a situation in which a user wanting to hear the song "Summertime" would be satisfied with just any recording of the song: the aria from Gershwin's opera Porgy and Bess; Billie Holiday's rendition with Dixieland-inspired band; Miles Davis's lyrical Harmon-muted trumpet over Gil Evans's lush winds; John Coltrane's upbeat modal honking tenor sax improvisation; Billy 
Stewart's stylized scat singing; Janis Joplin's gutsy and soulful vocals; or Fun Boy Three's very '80s new wave pop version. In the minds of users, each one of these recordings is distinct, and one cannot substitute for another. George Gershwin's original aria is simply a framework upon which new works are built by the individual recording artists.

Gershwin's "Summertime" may be an exception to the rule, considering Porgy and Bess's status as an American masterpiece, but popular music listeners often do not know who the songwriters of the music to which they listen are, even when the songwriters are extremely famous. To the popular music listener, the performer is the primary "creator" of the artwork. In 1973, Dolly Parton wrote and recorded the song "I Will Always Love You," which subsequently went to \#1 on the Billboard Hot Country charts twice, first for the original recording released in 1974 and again in 1982 when she re-recorded the song for the movie Best Little Whorehouse in Texas. Whitney Houston recorded "I Will Always Love You" as an R\&B ballad for the soundtrack to the 1992 movie The Bodyguard. This cover version was hugely successful, far surpassing the original by charting at the top of the Billboard Hot 100 for twenty weeks. "I Will Always Love You" has become one of Houston's signature songs, so much so that upon her untimely death in February 2012, her recording of the song shot up the Billboard charts again, all the way to \#3. In an interview published in Billboard magazine, Danny Nozell, Dolly Parton's manager, said, “One thing we found out from American Idol: most people don't know that Dolly Parton wrote 'I Will Always Love You."'9

As can be seen, there are quite marked differences between the classical music and popular music cultures from the point of view of the composers, performers, and users. It stands to reason that creators and users of metadata for these two traditions may expect them to be treated differently in the library catalog. While FRBR is quite suitable in modeling classical music, ${ }^{10}$ our conservative application mandating the composer/composition construction of AAPs for all musical works could be interpreted as cultural bias. As catalogers of high art music, Top 40 radio music, and everything in between, we should not let any such potential biases work to the detriment of the catalog data or the FRBR user tasks. 


\section{PROBLEMS FOR CATALOGERS}

A major argument against continuing with the current RDA practice as applied to popular music is the fact that constructing work AAPs according to this practice can sometimes be more burdensome than it is worth. One of the main reasons to create work AAPs is to uniquely identify works in the database, and presumably allow a catalog or discovery tool to assist users in identifying or finding resources that contain or are related to these works. One could argue that these "headings" should also be constructed to be comprehensible to users. If AAPs are not meaningful, they cannot act as effective shorthand for the works, thus undermining one of the benefits that AAPs bring in the first place.

North American music catalogers have been using a conservative interpretation of the FRBR/RDA concepts of work and creator in cataloging popular music, the work being the composed song and the creator being the songwriter. While the composer/composition construction of AAPs seems relatively straightforward, creating them for real-world popular music works is not always easy. Setting aside the already discussed philosophical arguments over what should be considered the work and who should be considered the creator, there are in fact some practical built-in barriers found in the popular music culture which impede such simple tasks as finding and determining the name of the creator.

Songwriting credits for popular music resources are not always easy to find, if available at all. As mentioned earlier, credits can be in a number of places within the resources, but almost never found on the front of the container - the so-called "album cover," the most visually identifiable aspect of a commercial recording. Sometimes songwriting credits are given on the back of the container or on the disc labels themselves. But just as often they are relegated to the inside of the liner notes, either listed with the song lyrics or on the last page of text. Songwriting credits are universally given in small print, the names often in abbreviated form (last name only or, if generous, with first initial). Taking the extra time to hunt for songwriting credits may not seem like a difficult task, but having to do so repeatedly severely impacts a cataloger's workload, not to mention the fact that the cataloger may then be required to match up the credited "S. Carter," for example, to the correct person in the authority file. 
Additionally, many performers who are also songwriters use their real names (rather than their better-known stage names) for songwriting credits. Should catalogers consider this a case of "individuals with more than one identity" as described in RDA 9.2.2.8? Strictly speaking, the alternate names correspond to different identities — the person's composer identity and performer identity — and according to RDA should be considered two separate entities with two separate AAPs (and two separate authority records), although this has generally not been the approach taken. Since much effort has been spent in distinguishing compositions (works) from performances (expressions) when cataloging music, it seems natural to continue this distinction and assign multiple identities for these individuals who create or contribute to these resources. Who is the creator of the song "Encore": "S. Carter" or "Jay-Z"? If catalogers choose "Jay-Z" for practical reasons, how is this practice compliant with RDA principles?

Many songs are written by teams of songwriters. The instructions for musical works with lyrics, libretto, text, etc. at RDA 6.28.1.2 say that AAPs for these works are constructed by combining the AAP representing the composer of the music with the preferred title of the work. For classical music, this instruction makes sense in most cases due to the status of the composer in this tradition. But there are other musical traditions in which artistry in lyric writing (or lyric improvisation) is an equally important, or even more important, aspect of the music, such as in rap music and ballad singing.

Of course, 6.28.1.2 can only apply when there is a clear distinction between composer and lyricist. Many popular music songs have songwriting credits in which each person's exact role is unnoted or unknown. In cases like this, the cataloger presumably follows the guidelines at RDA 6.27.1.3 which instruct her/him to use the collaborator "with principal responsibility" as the first part of the AAP. If roles and principal-ness are not indicated, the first listed collaborator is used, a practice which could possibly misrepresent the nature of the work when songwriting credits are given in an arbitrary order (which they often are). There are many songwriting teams, like Lennon \& McCartney and Jagger \& Richards, for which roles or principal-ness are not stated. In fact, some of these partnerships have agreements in which both individuals are credited for every song, even when only one person wrote the entire song. Choosing 
the first-named person of a songwriting duo to be a song's composer is thus an arbitrary simplification of a more nuanced truth.

What if the featured performer is also one of the collaborators who created a song? The Henry Bishop example in RDA 6.27.1.3 suggests that typographical prominence of a collaborator's name can indicate that entity's principal-ness. Does the prominence of a name on the resource's cover automatically promote this person/corporate body to "principal responsibility" for any of the songs contained therein? What if the performer wrote the lyrics but not the music, or is included in the songwriting credits only for contractual reasons, having contributed little to the actual compositions?

To further complicate matters, many songs are written by bands. Unfortunately, the method of crediting songwriting to musical groups is not standardized in the industry. In some cases, such as Duran Duran's first few albums, the actual band name is given as the songwriter: "All songs written by Duran Duran." This is a clear case of corporate body creatorship. But in other cases, the names of the individual band members are given, not the name of the band. The RDA glossary defines a corporate body as "an organization or group of persons and/or organizations that is identified by a particular name [writers' emphasis] and that acts, or may act, as a unit." If the songwriting is credited to all band members named individually, can/should a cataloger be expected to take the logical step to conclude that the corporate body (even though not "identified by a particular name" in the credits) is the creator of the work? The examples for Coldplay and Red Hot Chili Peppers under RDA 19.2.1.3 indicate that these corporate bodies are the creators of the works Parachutes and Stadium Arcadium, respectively. But close inspection of the songwriting credits in each of the actual albums shows that the individual band members' names are used, not the name of the band:

"All songs by Berryman/Buckland/Champion/Martin"

“All songs (C2006 and written by Anthony Kiedis, Flea, John Frusciante and Chad Smith"

What appear on the resources to be collaborative works are determined in RDA to actually be works by a single corporate creator by virtue of the bands' memberships. So during the cataloging process, the cataloger must do the math: 


\section{Berryman + Buckland + Champion + Martin $=$ Coldplay}

\section{Anthony Kiedis + Flea + John Frusciante + Chad Smith = Red Hot Chili Peppers}

However, membership of musical groups is often fluid, with members coming and going over time. (Please read the Wikipedia article "Red Hot Chili Peppers"11 to get a sense of the difficulty one could face in determining the membership of a band at a certain time.) Making the above determination in cataloging would require that catalogers know a band's specific membership at the time of a work's creation.

The above example opens the door to some further questions regarding the nature of corporate body-hood in RDA. What if a song is written by most, but not all, members of a band? Do four of five members acting "as a unit" to write a song equate to the whole corporate body creating the work, or does incomplete participation relegate that work to being a collaboration of the individuals? The presentation of the credits is somewhat of an arbitrary decision on the part of the band. While some bands attribute each song on an album to the specific member or members of the band who conceived of the lyrics and the most basic musical elements such as the chord progression and vocal melody, other bands, like Duran Duran, state that all songs were written by the entire band, as mentioned above. Does this mean that every member of the band had a hand in writing the lyrics, chord progression, and melody? Probably not. Instead, it probably means that once the basic framework for each song was established, all the members participated in filling it out with their own parts, deciding exactly which notes to play in guitar riffs, which rhythms to play on the drum set, etc. The bands who do attribute their songs to one or two individual members would likely have engaged in that same process.

In the opposite case, songs are sometimes written by all members of a band plus additional songwriters. Are the extra songwriters subsumed into the corporate body (acting as a unit), thus creating a work by a single creator? Or is the work a collaboration? What if the extra songwriter is closely associated with the group, such as a producer, manager, or past/future member? Since RDA's definition of corporate body is vague, one must also wonder how to interpret creatorship for an album on which all songs are composed variously by members of a band, as with the Beatles' album Abbey Road, which contains thirteen songs by Lennon \& McCartney, two by George Harrison, and one by Ringo Starr 
(credited as "Richard Starkey"). All four of these musicians were members of the Beatles when the album was created, so does one consider Abbey Road to be the "collective activity of a performing group as a whole" (a creation of the Beatles as a unit as described in RDA 19.2.1.1.1), or is this album a compilation of works by different band members working separately to create separate songs? The problematic situations outlined here are not far-fetched, but actually quite common in popular music, and in order to truly follow the composer/composition interpretation of FRBR, the cataloging communities who use RDA need to formally clarify these instructions and definitions.

\section{SONGS IN FRBR AND RDA}

While the overwhelming majority of library metadata for popular music audio recordings is at the album level, considering a song's place in the world of FRBR and RDA is nonetheless a valuable exercise for several reasons. (1) A song is a discernible unit of bibliographic content - a musical work, in RDAand therefore should be considered on its own terms. (2) In some cases, it is useful to create AAPs for the individual songs on a cataloged album, be it a typical album in a recording artist's output or a compilation of hit songs by various artists. (3) Consumers of popular music increasingly act in a song-based economy in which popular streaming and download services make individual songs available independently from their parent albums. (4) Individual songs receive detailed attention in popular online discographies and databases with which a library catalog should strive to align, especially as integration with these resources via Linked Data becomes a possibility. In addition, catalogers are increasingly being asked to process recordings in popular music special collections, such as Cornell's hip-hop and punk collections, which contain large numbers of 7" singles. ${ }^{12}$

We've discussed the prominence of the performer over songwriter in the popular music culture, readily witnessed in an ordinary pop single such as Miley Cyrus's "Wrecking Ball," written for her by a team of songwriters (Dr. Luke (Lukasz Gottwald), MoZella (Maureen McDonald), Stephan Moccio, Sacha Skarbek, and Cirkut (Henry Walter)). The prominence of Cyrus's name and image on resources containing the song demonstrates the fact that musical artists are often more closely associated with songs 
than songwriters, at least by users. Also notable are the plethora of cover songs that become closely associated with the cover artist (as exemplified by "Without You," "Proud Mary," and "I Will Always Love You"). But there is a host of other phenomena in the popular music realm that illustrate this point even more clearly, such as sampling, mashup, and parody. We will use examples of each of these to demonstrate how exclusion of the musical artist (performer, producer, etc.) from a song's AAP can be taken to the point of absurdity.

The technique of sampling recordings of earlier songs in new songs has been used heavily but not exclusively in hip hop music. ${ }^{13}$ The sampled song may be used to varying degrees in the new song, sometimes just adding a brief moment's reference to the sound of the earlier song, and other times providing the basis for the new song. Robin Thicke, before he became infamous for his song "Blurred Lines" with its copyright-infringing musical "feel," pushed sampling to the limit in his single "When I Get You Alone." Similar to Gounod overlaying his “Ave Maria” melody on a keyboard prelude by Bach, Thicke layers a newly composed vocal melody and amorous lyrics over the complete recording of Walter Murphy's instrumental disco classic “A Fifth of Beethoven.” Murphy's single is clearly a free adaptation of musical elements from Beethoven's fifth symphony, and therefore a new work. But with Thicke's song, things are not so clear-cut, especially given that at least some releases of this song give the credits as: "Written by Robin Thicke \& Walter Murphy... Composer/Arranger: Walter Murphy, BMI." What does one do when presented with information like this? Should the AAP be formulated honoring Murphy's composer credit on the packaging, even though his involvement likely didn't go beyond collecting royalties while his earlier track was sampled?

\section{Murphy, Walter, 1952- When I get you alone}

It is unlikely that this AAP would match a catalog user's expectations.

British producer Freelance Hellraiser's “A Stroke of Genius” is an incredibly clever mashup of the vocals from "Genie in a Bottle" by Christina Aguilera and the instrumentals from "Hard to Explain" by the Strokes. Obviously, a relationship exists between "A Stroke of Genius" and the two individual songs that it mashes up, and this relationship should be able to be modelled in FRBR, and in RDA using 
AAPs. But how? If we accept that "A Stroke of Genius" is a new work, then what are the works it is based on? Freelance Hellraiser's compositional materials in creating "A Stroke of Genius" were not chords, lyrics, and melodies; they were audio tracks. To assert a direct bibliographic relationship between the work "A Stroke of Genius" and the compositions "Hard to Explain" by Strokes frontman Julian Casablancas and "Genie in a Bottle" whose words and music are credited collectively to Steve Kipner, David Frank, and Pam Sheyne would be incongruous.

\section{Freelance Hellraiser. Stroke of genius \\ Based on (work): Casablancas, Julian. Hard to explain \\ Based on (work): Kipner, Steve. Genie in a bottle}

The parody songs of "Weird Al" Yankovic provide an entertaining lens through which to look at pop songs in FRBR and RDA. In songs such as "Eat It" (after the Michael Jackson song "Beat It"), "Like a Surgeon" (after Madonna's "Like a Virgin"), and more recently, "Word Crimes" (after "Blurred Lines," mentioned earlier), the basic musical content is essentially the same as that of the songs being parodied. Of course, the parodic lyrics create entirely new layers of meaning, so could these songs be considered new works? Unfortunately, RDA 6.28.1.2 thwarts this notion by privileging the composer of the music over the lyricist in constructing the AAP. Does that mean that "Eat It" and "Beat It" are the same work, to be represented by the same AAP?

\section{Jackson, Michael, 1958-2009. Beat it}

This is certainly not ideal, but it could be worse. Michael Jackson was both the composer and performer of "Beat It," but consider "Like a Surgeon," written by Billy Steinberg and Tom Kelly:

\section{Steinberg, Billy. Like a virgin}

This potential AAP for "Like a Surgeon" would be twice-removed from the song's musical artist, Weird Al, since Madonna did not actually write the song being parodied. People were setting new lyrics to existing music long before Weird $\mathrm{Al},{ }^{14}$ and there actually is an RDA instruction that accommodates works with new text and title (6.28.1.6). Oddly enough, though, this instruction is restricted to operas and other 
dramatic works and therefore does not account for non-dramatic works such as "Eat It" and "Like a Surgeon." Future revisions to RDA may rectify this, but it is not yet clear how.

Another phenomenon that occurs with some regularity in the popular music sphere is the coexistence of an "explicit" album version and a "clean" radio edit of a song. Usually, the offensive word or phrase is simply obscured in the radio edit, but in some cases, such as N.W.A.'s "Straight Outta Compton," entirely new vocal tracks are recorded with substituted "clean" lyrics. ${ }^{15}$ Although these lyrically different versions of songs do not approach the level of Weird Al's parody songs in creating new layers of meaning, they do beg us to think about where the line between expression and new work is drawn. Sometimes, though, lyrical differences between an explicit version and clean version of a song can affect the title of the song, and thus affect access via the song's AAP. Such is the case with CeeLo's song "F**k You" (as the title is usually stylized on resources containing the song) and its clean counterpart "Forget You," written by Bruno Mars, CeeLo Green, and Philip Lawrence. Presumably, the AAP for this song would be:

\section{Mars, Bruno, 1985- F**k you}

Using this AAP in connection with resources containing CeeLo's recording of the clean version would mean that neither the artist (CeeLo) nor the title ("Forget You") would be present in the AAP. There also exists a wordless, clucking-only rendition of this song by Camilla and the Chickens from the 2011 film The Muppets. Would the same AAP be used in connection with the soundtrack album, even though it bears the title "Forget You" in the track listing? If so, we'd wind up with Camilla and the Chickens as performers of an expression of a musical work called " $F^{* *} \mathrm{k}$ You."

Aided by the Internet as a platform for dissemination, musical artists (both professional and amateur) are boundless in their ability to put their own creative spin on existing musical materials. They make and upload videos of themselves singing songs in their bedrooms while accompanying themselves on only a guitar; they compete in televised talent contests, singing renditions of popular classics that may be fairly faithful to the original versions overall but inevitably introduce some degree of variation in their musical details; they record and sell new versions of songs popularized by earlier recording artists, 
sometimes transforming the musical content almost beyond recognition; they dream up ways to marry two totally unrelated songs and create something new; they change lyrics, they remove lyrics, they add lyrics where none existed previously, and they even substitute animal sounds for lyrics. Subjected to remakes, remixes, mashups, parodies, and other techniques, a pop song sometimes begins to resemble a folk song, in that it was composed a long time ago, and the public has forgotten who composed it, if they ever knew. What they do know and remember, however, is who performed it.

\section{ALBUMS IN FRBR AND RDA}

The above-mentioned issues are specific to creating AAPs for a single work. Most libraries, though, do not collect single popular music songs, but rather albums, considered "aggregates" in FRBR and "aggregate works" or "compilations" in RDA. Although it is not defined in the RDA Glossary, the term "compilation" is used pervasively in RDA chapter 6 in the instructions for recording titles and constructing AAPs for works. In these instructions, "compilation" is not used in the "best workout songs from the 1980s" sense, but refers to any work made up of other works - including, therefore, any standard album by a recording artist. We will now examine the implications of this, for standard albums as well as a few unusual cases.

Under our current RDA practice, a person or corporate body must have songwriting credit for all works contained in the album in order for its AAP to be used as part of the AAP representing the album. For example, Stevie Wonder wrote all of the songs on his album Innervisions: "All tunes are written by Stevie Wonder." Since he is clearly the creator of each individual song, or musical work, he is also the creator of the album, or compilation of musical works. The AAP for this album would be:

\section{Wonder, Stevie. Innervisions}

While Innervisions lists the songwriting credits collectively for the entire album, many albums list songwriting credits separately for each song contained therein, and in order determine whether a creator merits inclusion in the AAP, the cataloger must fastidiously check each song. What if the cataloger determines that a single person or musical group has songwriting credit on each and every song 
on an album, but select songs have additional songwriters (i.e. are collaborations)? On her chart-smashing album 1989, Taylor Swift wrote every song, but twelve of thirteen were written with other songwriters. Should the cataloger consider this album to be the work of the single entity that unifies the whole?

\section{Swift, Taylor, 1989- 1989}

Or is it a compilation of works by different sets of collaborators (despite the fact that a single person collaborates on each and every work)?

\section{9 (2014)}

If it is determined that a single entity does not have songwriting credit for all the songs on an album, then the AAP is constructed using the preferred title without a creator (what AACR2 called "Main entry under title"). When considering popular music resources, a good portion of albums would fall in this category of "compilations of works by different persons, families, or corporate bodies" because all songs are not composed by the same entity. Miley Cyrus's album Bangerz is a "compilation" of songs with diffuse authorship. Since no single person is the composer of all the songs, no single person can be said to be the creator of the album, and the AAP would be simply:

\section{Bangerz}

This is a major change from AACR2 that affects even the most typical commercial studio albums. AAPs for innumerable albums recorded by some of the most well-known popular musicians of the 20th and 21st centuries would be constructed title-first: Miley Cyrus, Frank Sinatra, Elvis Presley, Aretha Franklin, Barbra Streisand, Britney Spears, the Beatles, the Jackson Five, and countless other pop, jazz, folk, gospel, country, and blues musicians.

AAPs for these types of compilations often require additional qualifiers to make them unique, a requirement set forth in RDA 6.27.1.9. The LC-PCC PS 6.27.1.9 takes this a step further by saying that these AAPs must not conflict with that for any other work in the database in which the cataloger works. (For most U.S. catalogers doing original cataloging, this would be the OCLC database.) Since there are a number of compilations with short, oft-used, generic titles such as "Live," "Greatest hits," "Christmas Album," or even "Elvis," checking the database for conflict is problematic and impossibly time- 
consuming. A title browse performed in the OCLC database for "Beatles" results in well over 2500 bibliographic records. Attempting to spot check for conflicts through trial and error (keyword searching a generic title and possible qualifiers) would ignore the reciprocal aim of collocating all publications containing the same work with a common AAP. An additional difficulty is the fact that the OCLC database (and local catalogs) contains not only RDA bibliographic records, but also those created according to other standards such as AACR2. Is a cataloger expected to mentally re-catalog these resources in order to check for theoretical duplicate AAPs? On the other hand, ignoring these bibliographic records created using AACR2 would undercut the potential of the AAPs.

RDA 6.27.1.9 allows the following qualifiers as "additions to access points representing works": form of work; date of work; place of origin of work; or another distinguishing characteristic of the work. The LC-PCC PS at 6.27.1.9 specifies further: "corporate body; date of publication; descriptive data elements, e.g., edition statement; place of publication." Once these four choices are exhausted ("If none of these qualifiers is appropriate...") and the AAP is still not unique, then the PS allows for the use of "any word(s) that will serve to distinguish the one work from the other." Trying to find an appropriate qualifier to make the AAP simultaneously unique and meaningful can be quite a challenge.

The FRBR/RDA concept of aggregates is especially problematic when we consider the relatively recent trend of popular music artists recording cover versions of existing albums. Pink Floyd's landmark album Dark Side of the Moon has inspired a number of track-for-track remakes, including the Squirrels' The Not-So-Bright Side of the Moon, Easy Star All-Stars' Dub Side of the Moon and Dubber Side of the Moon, the Flaming Lips' The Flaming Lips and Stardeath and White Dwarfs with Henry Rollins and Peaches Doing The Dark Side of the Moon, and others. The Beatles' equally famous Sgt. Pepper's Lonely Hearts Club Band has also had the honor of being remade by both Easy Star All-Stars and the Flaming Lips, as Easy Star's Lonely Hearts Dub Band and With a Little Help from My Fwends, respectively.

Clearly, the artists responsible for these reimaginings enjoy getting creative with the titles of the albums; this should be a signal to catalogers of the significant originality of the music, along with the fact 
that the cover version sometimes crosses over into a new genre, such as reggae or dub. Yet a strict interpretation of FRBR and RDA could lead to these albums merely being considered to be compilations of expressions of existing musical works - which happen to be identical in content and sequence to the original albums, which are in turn considered compilations by virtue of the diversity of authorship of their songs. For example, the AAP for Pink Floyd's album as a work could be:

\section{Dark side of the moon (Pink Floyd (Musical group) : 1973)}

This could mean that the AAP for both of Easy Star All-Stars' albums would also be:

\section{Dark side of the moon (Pink Floyd (Musical group) : 1973)}

Things would play out similarly at the song level, with the same work-level AAPs being used for both Pink Floyd's and Easy Star All-Stars' performances. For example:

\section{Waters, Roger. Money}

None of the above possibilities is particularly appealing, especially since the titles by which Easy Star All-Stars' albums are known to fans may not even make an appearance in the album-level AAPs, and Pink Floyd would not make an appearance in any of the song-level AAPs.

Another early $21^{\text {st }}$-century trend is the mashup, already discussed at the song level, but also occurring at the album level. In 1968, the Beatles released a self-titled album that became known as The White Album (but is listed as The Beatles in the U.S. national authority file as well as various discographies). In 2003, rapper Jay-Z released The Black Album, prompting DJ Danger Mouse to create The Grey Album, an entire album of mashups combining the two, in 2004. Danger Mouse's compositional materials in creating the selections on The Grey Album were the audio tracks taken from The White Album and The Black Album — not the actual compositions by the members of the Beatles (variously, Lennon \& McCartney, Harrison, and Starkey) or by S. Carter (i.e. Jay-Z) and his collaborators. So, how should the abundant relationships that exist be articulated? For the album, we might have:

\section{Danger Mouse (Musician). Grey album \\ Based on (work): Beatles (Beatles : 1968) \\ Based on (work): Black album (2003)}


For "Encore," a song from The Grey Album, we might have:

\section{Danger Mouse (Musician). Encore \\ Based on (work): Jay-Z, 1969- Encore \\ Based on (work): Lennon, John, 1940-1980. Glass onion \\ Based on (work): Harrison, George, 1943-2001. Savoy truffle}

An irksome incongruity results when Danger Mouse is eligible for creatorship but the Beatles (and Jay-Z, at the album level, because the first track "Interlude" is uncredited) are not.

\section{A PROPOSED SOLUTION}

Thinking through all of the above-mentioned issues while cataloging popular music can be prohibitively time-consuming and complicated, and when the AAPs for these works are finally constructed, they are often not very useful for humans in finding or identifying popular music resources. With expanded workloads, shrinking budgets, and fewer music cataloging specialists, one wonders how long our current practice can be sustainable. The authors here propose a solution that would allow catalogers to create intuitive AAPs for popular music works that better correspond with most users' understanding of popular music's "bibliographic universe." While we don't expect all complicated issues to be resolved, the majority of popular music resources' AAPs will be much more easily constructed.

In our proposed model, performances of popular music songs are considered new works based on the underlying composed song. Performers (or in some cases music producers) are the creators of these new works. The original composed songs also exist as separate related works, the songwriters being the creators of those. Performed songs are adaptations of the original composed songs, to which are added the performers' and producers' artistic styles and creative choices. The AAP for the performed song represents not any specific performance, but the general artistic vision that the musical artist applies to all performances of that song, using that general set of creative decisions - an abstract entity that exists only in the mind of the creator. Variant versions or performances of that song by that performer are expressions of that abstract work. 
The possibility remains that a single musical artist could create another entirely new version of a song based on very different creative decisions, and this can be considered another new work with a different AAP. For example, the two versions of the song "Don't Stand So Close to Me" released by the Police in 1980 and 1986, or the two versions of Joni Mitchell's "Both Sides, Now" from 1969 and 2000, should probably be considered independent works. The musical artists drastically changed the styles, textures, instrumentation, tempo, and even keys of these songs from one recording to the other.

\section{Police (Musical group). Don't stand so close to me \\ Police (Musical group). Don't stand so close to me '86}

\section{Mitchell, Joni. Both sides, now}

\section{Mitchell, Joni. Both sides, now (2000)}

RDA AAPs for performed popular music songs would be constructed by combining the AAP of the entity presented on the resource as having principal responsibility (usually a performer, but sometimes a producer) with the preferred title chosen by applying guidelines at RDA 6.2.2 to that musical artist's resources (usually the title of the song as it appears on the resource). Since popular music is very often a collaborative effort of multiple performers and producers, the RDA instructions in 6.27.1.3 can help in determining whose name is used in the AAP for the work. When a song is recorded by different musicians, each musical artist's version of a song would have an AAP containing the name of the artist (or whoever has principal responsibility), not the composer.

In popular music, there is not a strict dichotomy between songwriter and performer; some situations are quite subtle. For example, "Uptown Funk" is presented as a song by Mark Ronson, a producer, and included on Ronson's album Uptown Special, but Bruno Mars is the featured performer. The songwriting credits on at least one CD single are as follows (in small print): "Composed \& written by: Bruno Mars, Jeff Bhasker, Mark Ronson and Philip Lawrence.” Sometimes it's impossible to know, when multiple songwriters are involved, which one if any had primary songwriting responsibility, nor should a cataloger be burdened with having to determine this. There may or may not be any significance to the order in which songwriters are listed, and the sequence may vary from one source to another or 
even in separate areas of a single resource. A pragmatic course of action in a case like this would be to consider Mark Ronson the creator of the song "Uptown Funk" based on the prominence of his name on the resource. Bruno Mars could be considered a collaborator, also based on the prominence of his name. Accepting the evidence at hand brings more consistency and efficiency to cataloging and respects how the artists themselves present their work.

As stated above, the original composed work still exists and could have an AAP of its own (composer/composition) if deemed important or necessary by the cataloger. Relationships between the recorded song and the original composed song can be made as 5xx references in authority records. Also, significant musical borrowing, such as sampling, parody, remixes, mashups, and borrowed melodies or lyrics, can also be noted in authority records. Of course, the AAPs for other people or musical groups collaborating in the recording can be cross-referenced in 4xx authority fields if the resources present them as having major significance to the creation of the work.

\section{Creedence Clearwater Revival. Proud Mary Based on (work): Fogerty, John. Proud Mary}

\section{Ike \& Tina Turner Revue. Proud Mary Based on (work): Fogerty, John. Proud Mary}

All versions of John Fogerty's song can still be collocated in the authority file (or for those catalogs which lack one, at least through an exact title keyword search):

\section{Fogerty, John. Proud Mary}

see also:

\section{Creedence Clearwater Revival. Proud Mary Ike \& Tina Turner Revue. Proud Mary$$
\text { Presley, Elvis. Proud Mary }
$$$$
\text { Ohio Players (Musical group). Proud Mary }
$$ Ventures (Musical group). Proud Mary}

Since popular music works would be an abstract entity, the actual performances or recorded versions of the musical artist's abstract song would be considered FRBR expressions. Assuming that they do not stray too far from the original recording, these expressions would include alternate takes, live recordings, remixes, radio edits, etc. By assigning the performance and capture characteristics to FRBR's 
expression entity, this solution harmonizes with FRBR's treatment of classical music. In many cases, popular music works exist only in a single recorded version; in others, multiple versions may exist but are similar enough to be considered roughly equivalent by users. These cases would likely only require a single AAP for the work. But the cataloger could choose to differentiate between variant expressions of a song if deemed important: album version and radio edit; the original version and a remix; various studio takes; recorded live performances. At the 2015 Grammy Awards, the Record of the Year designation was awarded to Sam Smith's "Stay with Me." The Academy, however, chose a very specific version of the song — not the original album version of the song, but the "Darkchild version," which is a remix produced by Rodney "Darkchild" Jerkins. By applying expression elements to the work AAP, one could distinguish the Darkchild mix of the song yet still maintain the concept of work collocating all versions of "Stay with Me" by Sam Smith.

While albums are the most common type of popular music resource held by libraries, conceptually they are not an easy type of resource to fit into the FRBR model. Our current interpretation of RDA considers albums simply as collections of songs which happen to be issued together. But in its final report ${ }^{16}$ of 2011, IFLA's Working Group on Aggregates acknowledged that the concept of aggregates was given rather vague treatment in the original FRBR report and that for some resources the act of selecting and arranging expressions into an aggregate requires intellectual contribution which should be reflected as responsibility at the work level. The simple model of the aggregate work does not always reflect real-life bibliographic resources.

In the case of studio albums, the featured musical artist not only selects and arranges songs into an aggregate, but actually creates a single unified work, each song contributing to the album's overall narrative flow, style, mood, and creative vision. While simplistically they could be considered just collections of songs, popular music studio albums contain much added artistic content. The authors believe that albums for which the featured musical artist made creative decisions should be treated as works in their own right, the musical artist's abstract artistic vision of collecting and recording a set of songs to create a unified whole. AAPs for albums would follow the same method of construction as 
described above for songs: use the name of the musical artist presented as having principal responsibility and the title as it appears on the resource. Alternate versions of the album, including variant geographical releases, remastered editions, and those with bonus content, would be variant expressions of the abstract album work. In our model, the songs contained on the album are independent of the album work, and the album and songs have work AAPs independent from one another.

By applying this solution, creating AAPs using information presented prominently on the resources themselves, the cataloger will save time and effort, while creating AAPs that better accommodate user needs rather than work against them. The key here is not over thinking who-is-what in FRBR terms, but simply considering the information on the resource at face value, as a user usually would. In fact, RDA 0.4.3.6 reinforces the idea that the presentation of principal responsibility may be more important than the actual responsibility.

The data recording relationships between a resource and a person, family, or corporate body associated with that resource should reflect attributions of responsibility, whether these attributions are accurate or not. Attributions of responsibility can be found either in the resource itself or in reference sources. ${ }^{17}$

Using the information as presented on the resource would also solve the dissonance felt by users when they see a song attributed to a name which they do not recognize. Under the current cataloging practice, most of the songs and albums by the band the Village People would have AAPs beginning with the name "Morali, Jacques," the band's producer, manager, and principal songwriter. An even more confusing situation is when the name is one that users do recognize, but not the name of the musical artist they seek. Aretha Franklin's signature song "Respect" was written by Otis Redding; while a famous performer himself who also recorded this song, his version holds nothing on the version by the Queen of Soul. User access issues can also occur when a cover version of a song is released under a different title from the original song. Barry Manilow's first number one hit and gold single "Mandy" was co-written and recorded by Scott English several years earlier under its original title "Brandy." Retaining the original title in an AAP would definitely be detrimental to the FRBR user tasks. 
In his article "Composing in Real Time: Jazz Performances as 'Works' in the FRBR Model,"18 Raymond Schmidt compares two alternative methods of creating AAPs for recorded jazz performances, interpreting the concept of FRBR work at two different levels: the first at the level of the composition; the second at the level of a specific performer's recorded improvisation on the composition. Our proposed solution takes a middle ground between Schmidt's two methods. The example of Art Tatum's recordings of the song "Tea for Two" could be represented by the AAP:

\section{Tatum, Art, 1910-1956. Tea for two}

Based on (work): Youmans, Vincent, 1898-1946. No, no, Nanette. Tea for two

This work AAP represents all of Tatum's solo piano performances. The two Art Tatum studio recordings of the song would be expressions, as well as any other recorded live performances. While these two recordings are quite different, Tatum's personal improvisatory style is a major unifying component.

\section{Tatum, Art, 1910-1956. Tea for two (1933)}

\section{Tatum, Art, 1910-1956. Tea for two (1939)}

In his article "The Definition of the Work Entity for Pieces of Recorded Sound," 19 Christopher Holden discusses the topic of how certain types of audio recordings could be FRBR works. He primarily focuses on the creative role of music producers, audio engineers, and creators of recording-based compositions and explores the possibility of making room for these individuals and their distinct recordings of the same performances at the work level. ${ }^{20}$ While we don't propose making such a distinction, the variety of ideas generated by Schmidt and Holden (and now us) demonstrate a dissatisfaction in our community with FRBR's application to popular music and a yearning to recognize the agents involved in making popular music in a manner befitting their contributions.

The argument can and will be made that music catalogers in North America have not generally created AAPs for popular music resources (at the album level, and almost never at the song level). The BIBFRAME AV Modeling Study explains:

Currently, cataloging practices for this type of content focus more on describing publications by creating records which represent the aggregation of the tracks, rather than 
on the individual tracks of a sound recording. Works realized on tracks are marginally described in such records, generally only in a contents note. Since information on popular music composers and lyricists is often incomplete or missing from liner notes, and because the cataloging time costs associated with researching and creating authority records for all such entities associated with each track on a given recording are relatively high, most popular music composers and lyricists are not individually traced in catalog records. ${ }^{21}$

However, looking toward the future, in order to integrate library catalog metadata into the Semantic Web of Linked Data, we must consider increasing the use of AAPs in bibliographic records to accommodate identifiers for all bibliographically related entities, not only for those entities who create a work but also the works themselves. Without the ability to link bibliographic data to identifiers, music metadata created by libraries will become further divorced from music metadata used elsewhere on the Internet.

At this point, we will revisit examples of songs and albums discussed in earlier sections of the article and show how satisfactory AAPs would be formulated in our proposed model. In the examples that follow, an AAP for a source work will be indented immediately beneath the derivative work.

Robin Thicke's song that samples Walter Murphy's disco single that is based on Beethoven's symphony could be represented like this:

\section{Thicke, Robin, 1977- When I get you alone}

Based on (work): Murphy, Walter, 1952- Fifth of Beethoven Based on (work): Beethoven, Ludwig van, 1770-1827. Symphonies, no. 5, op. 67, C minor

We can also solve the incongruity of asserting a direct relationship between the mashup work "A Stroke of Genius" and the compositions "Hard to Explain" and "Genie in a Bottle." If the singles by the Strokes and Christina Aguilera are considered works in their own right, then the relationships drawn between Freelance Hellraiser's audio work and the two audio works it uses as raw material is a satisfying one that realistically depicts the creative process involved in creating a mashup.

\section{Freelance Hellraiser. Stroke of genius}




\section{Based on (work): Strokes (Musical group). Hard to explain Based on (work): Casablancas, Julian. Hard to explain \\ Based on (work): Aguilera, Christina, 1980- Genie in a bottle Based on (work): Kipner, Steve. Genie in a bottle}

The AAPs for Danger Mouse's The Grey Album and the albums that inspired it would also be fair and intuitive:

\section{Danger Mouse (Musician). Grey album \\ Based on (work): Jay-Z, 1969- Black album \\ Based on (work): Beatles. Beatles (1968)}

Instead of puzzling over who to call the composer of Weird Al's "Like a Surgeon," catalogers could simply choose the most prominently presented entity to be the creator, which, in this case, would also neatly account for Weird Al's creator-level role in devising new lyrics:

\section{Yankovic, Al, 1959- Like a surgeon}

Based on (work): Madonna, 1958- Like a virgin

Based on (work): Steinberg, Billy. Like a virgin

And instead of calling Camilla and the Chickens the performer of an expression of a musical work called "F**k You," catalogers could call them the creator of their rendition of "Forget You," using judgement in whether or not to reference CeeLo's singles and/or the composition by Bruno Mars. This may be novelty music, but it's still music, and it should be represented in a sensible way in library catalogs.

Earlier, we presented a bleak scenario for constructing AAPs for two Easy Star All-Stars albums, based on a strict interpretation of FRBR and RDA in which these albums could be considered compilations of expressions of existing musical works which happen to match those in Pink Floyd's Dark Side of the Moon. We would do well to take a step back from such an interpretation and acknowledge that the most meaningful relationship in this situation exists between the cover artist's album and the original artist's album - that is, these cover albums are more than mere performances (expressions) of compositions (works) written by the various individual members of Pink Floyd. They are highly original interpretations of the definitive recording of the album as a whole:

Easy Star All-Stars (Musical group). Dub side of the moon

Based on (work): Pink Floyd (Musical group). Dark side of the moon 


\section{Easy Star All-Stars (Musical group). Dubber side of the moon \\ Based on (work): Pink Floyd (Musical group). Dark side of the moon (and maybe also)}

Based on (work): Easy Star All-Stars (Musical group). Dub side of the moon

\section{CONCLUSION}

The solutions suggested here are both principled and practical. They align well with RDA's stated objectives of responsiveness to user needs, cost efficiency, and continuity (see RDA 0.4.2) as well as its principles of representation and attribution (see RDA 0.4.3). On the horizon are the further development and eventual implementation of BIBFRAME (the Linked Data model intended to replace the almost 50year-old MARC standard for encoding bibliographic data) and the completion of FRBR-LRM (FRBRLibrary Reference Model, which will consolidate FRBR for bibliographic data, FRAD for authority data, and FRSAD for subject authority data). ${ }^{22}$

Since RDA is based on FRBR, RDA will necessarily change once FRBR-LRM is published. And, since BIBFRAME is still in development, the BIBFRAME AV Modeling Study may influence the final product. It is worth noting that in its current state, BIBFRAME does not even make a distinction between works and expressions, so it may not even be possible to consider a recording artist's rendition of a song to be an expression of the composed work. These would likely be input as related works in BIBFRAME. ${ }^{23}$ One would hope that the players involved in these game-changing efforts will seek to ensure compatibility between FRBR-LRM/RDA and BIBFRAME while maintaining a commitment to the objectives and principles laid out in RDA and augmenting the capacity of library data to integrate with outside sources of Linked Data.

Even before these models are fully developed over the coming years, we need look no further than the visible Web for a model of how best to configure our popular music data. The conventional wisdom is that information seekers by and large start their research on an Internet search engine. Who can blame them, when there are robust, freely accessible online discographical databases, such as AllMusic ${ }^{24}$ and Discogs, ${ }^{25}$ and even the search engines themselves are getting in the game of presenting the searcher 
with structured data to navigate instead of just a list of links to Web pages? All of these tools organize their principal entries for songs and albums around performers and not songwriters. Standard citation practice across various style guides also privileges the recording artist for recordings of popular music.

The old paradigm of popular music albums being filed by recording artist in record stores and public libraries to facilitate patron browsing is not dead. Those brick-and-mortar institutions still provide highly valued browsability of sought-after tangible resources, and in fact, we have fielded numerous questions from catalogers in public libraries expressing concern about how AAPs for popular music albums might adversely affect classification and advised them that they can carry on classifying their CDs by artist regardless of how the AAPs might be formulated. The browsing experience desired by their users is replicated virtually in library catalogs that offer this functionality, ${ }^{26}$ which is coming back in fashion in some quarters after falling by the wayside within the last decade as many libraries implemented new discovery systems that abandoned this traditional search mode in favor of faceted browsing. Beatles albums, rather than being scattered across the alphabet, as suggested in the introduction, can file together in an author-title index:

Beatles. Abbey Road

Beatles. Hard day's night

Beatles. Help!

Beatles. Let it be

Beatles. Meet the Beatles!

Beatles. Revolver

Beatles. Rubber soul

Beatles. With the Beatles

This can only happen if we have the good sense to structure the AAPs this way. ${ }^{27}$

Many of the examples of songs and albums discussed in this article were chosen for their obvious originality. In these examples, it seems indisputable that a new work —an adaptation —is created. But catalogers shouldn't have to make such a value judgment about the degree of originality involved in the 
music they are cataloging. There often isn't sufficient time or expertise to make such judgments, and furthermore, such subjective work would lead catalogers to different conclusions. This is a perilous proposition in the context of a niche in the bibliographic universe where variant manifestations (reissues, remastered releases, deluxe editions, versions with bonus content, etc.) abound. The consistency that is so crucial in a shared cataloging environment would be lost as we inevitably fail in our efforts to bring order to the huge body of legacy data as newly created data for similar content merges with it, while arguably nothing is gained.

The job of catalogers is not to build a database of rights holders (although rights information does have its place). Our job is to build a bibliographic database intended to represent the world's creative output and reflect the way that bibliographic resources and the agents responsible for their existence present themselves. When we are beset by practically unanswerable questions of creatorship, wouldn't we be wise to short-circuit the unsolvable philosophical quandaries that arise and choose a simpler approach? After all, it's pop music — music for the masses. Cataloging it should not be rocket science.

The authors do not wish to diminish the importance of songwriters, since without them, there would be no songs to sing. We're also not saying that performers of the very lightly scripted aleatoric music of John Cage and others in the classical music tradition are any less creative than performers of popular music. We're simply advocating looking at those performers through whichever lens is contextually appropriate. Our proposal has nothing to do with the relative worth of composers/songwriters and performing/recording artists, but rather aims to make the best possible use of available resources (both human resources and information resources) and harmonize our work with that of the broader information community of which we are a part. While it may be technically true that simply linking the identifiers underlying our AAPs with identifiers used in outside sources such as LinkedBrainz $^{28}$ and DBpedia ${ }^{29}$ will help to disambiguate between (and collocate) entities - think of the Semantic Web rallying cry of "things, not strings"—we still need labels for those "things" that look nice and are comprehensible to our target audience. We need them now, and we will continue to need them in the Linked Data future. 
RDA development, particularly in specialized areas such as music, has been a lengthy process, and it is still ongoing. (For background information on FRBR and RDA development in a music context, see Glennan, ${ }^{30}$ Harden, ${ }^{31}$ and Iseminger. ${ }^{32}$ ) In the coming year, the authors will likely be involved in a formal examination of RDA with an eye to massaging the instructions and appendices to more readily accommodate popular music, under the purview of the JSC Music Working Group ${ }^{33}$ and other representative organizations in the music cataloging community. The primary change we hope to see in RDA is some mechanism in chapter 6 that allows the cataloger to exercise deference to various musical cultures. Secondarily, we hope to coin new relationship designators at the work level in appendices I and $\mathrm{J}$ for musical artists and their renditions of songs. It is hoped that the present piece will promote discussion and pave the way for change.

1 Resource Description and Access (Chicago: American Library Association; Ottawa: Canadian Library Association; London: Chartered Institute of Library and Information Professionals (CILIP), 2010-). In RDA Toolkit: http://www.rdatoolkit.org.

${ }^{2}$ Functional Requirements for Bibliographic Records: Final Report. Approved by the Standing Committee of the IFLA Cataloguing Section on September 1997 as amended and corrected through February 2009. http://www.ifla.org/files/assets/cataloguing/frbr/frbr_2008.pdf.

${ }_{3}^{3}$ Anglo-American Cataloguing Rules, 2nd ed., 2002 rev. (Chicago: American Library Association, 2003).

${ }^{4}$ Functional Requirements for Bibliographic Records, 17.

${ }^{5}$ Ibid. 19

${ }^{6}$ Ibid. 17

${ }^{7}$ Kara Van Malssen, "BIBFRAME AV Modeling Study: Defining a Flexible Model for Description of Audiovisual Resources," (May 15, 2014). http://www.loc.gov/bibframe/pdf/av-appendix-a.pdf.

${ }^{8}$ Ibid. Appendix A, 2.

${ }^{9}$ Ray Wadell, "The Importance of Being Dolly," Billboard, April 26, 2008, 31.

${ }^{10}$ Sherry L. Vellucci, "FRBR and Music," in Understanding FRBR: What It Is and How It Will Affect Our Retrieval Tools, ed. Arlene G. Taylor (Westport, CT: Libraries Unlimited, 2007), 131-151.

${ }^{11}$ See https://en.wikipedia.org/wiki/Red_Hot_Chili_Peppers.

${ }^{12}$ For more information about the Cornell hip-hop collection, see http://rmc.library.cornell.edu/hiphop/.

${ }^{13}$ For some examples, see http://www.whosampled.com/.

${ }^{14}$ See "The Star-Spangled Banner."

15 "Straight outta Compton / here's a crazy brother named Ice Cube / from a stupid dope gang with an attitude..."

${ }^{16}$ IFLA Working Group on Aggregates, "Final Report of the Working Group on Aggregates," (September 12, 2011). http://www.ifla.org/files/assets/cataloguing/frbrrg/AggregatesFinalReport.pdf.

${ }^{17}$ Resource Description and Access, 0.4.3.6.

${ }^{18}$ Raymond Schmidt, "Composing in Real Time: Jazz Performances as 'Works' in the FRBR Model," Cataloging \& Classification Quarterly 50, no. 5-7 (2012), 653-669.

19 Christopher Holden, "The Definition of the Work Entity for Pieces of Recorded Sound," Cataloging \& Classification Quarterly 53, no. 8 (2015), 873-894.

${ }^{20}$ For an exploration of ideas of authorship with respect to popular music performers, producers, recording studios, record labels, etc., see: Ron Moy, Authorship Roles in Popular Music: Issues and Debates (New York: Routledge, 2015).

${ }^{21}$ Van Malssen, Appendix A, 3. 
${ }^{22}$ Pat Riva and Maja Zumer, "Introducing the FRBR Library Reference Model" (paper, IFLA World Library and Information Congress, Cape Town, South Africa, August 20, 2015). http://library.ifla.org/1084/1/207-riva-en.pdf.

${ }^{23}$ Similarly, OCLC's parallel efforts to model works using Schema.org and BiblioGraph result in the conflation of FRBR's work and expression concepts into schema:CreativeWork. See: Carol Jean Godby, Shenghui Wang, and Jeffrey K. Mixter, Library Linked Data in the Cloud: OCLC's Experiments with New Models of Resource Description (San Rafael, CA: Morgan \& Claypool Publishers, 2015).

${ }^{24}$ See http://www.allmusic.com/.

${ }^{25}$ See http://www.discogs.com/.

${ }^{26}$ See catalogs of Cornell University Library (https://newcatalog.library.cornell.edu/) and the University of Chicago Library (https://catalog.lib.uchicago.edu/) for examples.

${ }^{27}$ The authority file already contains AACR2 authority records for most of these Beatles albums. One additional advantage of constructing RDA AAPs in our suggested manner would be a general harmonization with AACR2 legacy records.

${ }^{28}$ See http://linkedbrainz.org/.

${ }^{29}$ See http://wiki.dbpedia.org/.

${ }^{30}$ Kathryn P. Glennan, "The Development of Resource Description \& Access and Its Impact on Music Materials," Notes 68, no. 3 (2012), 526-534.

${ }^{31}$ Jean Harden, "Cataloguing in 2012: On the Cusp of RDA," Fontes Artis Musicae 59, no. 3 (2012), 249-256.

32 Damian Iseminger, "Works and Expressions in RDA: Problems and Solutions," in Directions in Music Cataloging, ed. Peter H. Lisius and Richard Griscom (Middleton, WI: Music Library Association and A-R Editions, 2012), 43-62.

${ }^{33}$ See http://rda-jsc.org/workinggroups. 